\title{
GENERATING FUNCTIONS OF JACOBI AND RELATED POLYNOMIALS ${ }^{1}$
}

\section{FRED BRAFMAN}

1. Introduction. This paper will give apparently new generating functions for Jacobi, ultraspherical, Legendre, and Hermite polynomials and a few results which follow from these generating functions. Most of the generating functions to be obtained here involve an arbitrary parameter, so that a generating function is obtained for each choice of the parameter.

Since authors are not uniform in notation, explicit expressions for these polynomials are included for convenience. In hypergeometric notation, Jacobi polynomials may be represented by

$$
P_{n}^{(\alpha, \beta)}(x)=\frac{(1+\alpha)_{n}}{n !}{ }_{2} F_{1}\left[\begin{array}{r}
-n, 1+\alpha+\beta+n ; \\
1+\alpha ;
\end{array}(1-x) / 2\right]
$$

and Hermite polynomials by

$$
H_{n}(x)=(2 x)^{n}{ }_{2} F_{0}\left(-n / 2,(-n+1) / 2 ;-;-1 / x^{2}\right) .
$$

The symbol $P_{n}^{(\alpha, \alpha)}(x)$ will indicate an ultraspherical polynomial, obtained by placing $\beta=\alpha$ in (1). By placing $\beta=\alpha=0$ in equation (1) there result the Legendre polynomials $P_{n}(x)$ :

$$
P_{n}(x)={ }_{2} F_{1}(-n, 1+n ; 1 ;(1-x) / 2) .
$$

Frequent use will be made of the factorial notation:

$$
\begin{aligned}
& (a)_{n}=\frac{\Gamma(n+a)}{\Gamma(a)}=a \cdot(a+1) \cdots(a+n-1) \text { for integral } n, \\
& (a)_{0}=1 \text { for } a \neq 0 .
\end{aligned}
$$

2. Jacobi polynomials. Definition (1) for Jacobi polynomials can be transformed into: ${ }^{2}$

$$
P_{n}^{(\alpha, \beta)}(x)=\frac{(1+\alpha)_{n}}{n !}\left(\frac{1+x}{2}\right)_{2}^{n} F_{1}\left(-n,-n-\beta ; 1+\alpha ; \frac{x-1}{x+1}\right),
$$

Received by the editors December 20, 1950.

1 This paper is a condensation of parts of the author's doctoral dissertation, Generating functions of Jacobi and related polynomials, written at the University of Michigan, 1950, under the direction of Professor E. D. Rainville, to whom grateful acknowledgment is hereby made. The dissertation was written while the author held a predoctoral fellowship from the United States Atomic Energy Commission.

See G. Szegö, Orthogonal polynomials, Amer. Math. Soc. Colloquium Publications, vol. 23,1939 , p. 67. 
which expands to

(5) $P_{n}^{(\alpha, \beta)}(x)=\frac{(1+\alpha)_{n}(1+\beta)_{n}}{2^{n}} \sum_{k=0}^{n} \frac{(x-1)^{k}(x+1)^{n-k}}{(n-k) ! k !(1+\alpha)_{k}(1+\beta)_{n-k}}$.

From (5) it follows that:

$$
\begin{aligned}
& \sum_{n=0}^{\infty} \frac{(a)_{n}(1+\alpha+\beta-a)_{n}}{(1+\alpha)_{n}(1+\beta)_{n}} P_{n}^{(\alpha, \beta)}(x) t^{n} \\
&=\sum_{n=0}^{\infty} \sum_{k=0}^{n} \frac{(a)_{n}(1+\alpha+\beta-a)_{n}(x-1)^{k}(x+1)^{n-k}(t / 2)^{n}}{(n-k) ! k !(1+\alpha)_{k}(1+\beta)_{n-k}}
\end{aligned}
$$

The right-hand side of (6) transforms to

$$
\sum_{k=0}^{\infty} \sum_{n=0}^{\infty} \frac{(a)_{n+k}(1+\alpha+\beta-a)_{n+k}(x-1)^{k}(x+1)^{n}(t / 2)^{n+k}}{n ! k !(1+\alpha)_{k}(1+\beta)_{n}}
$$

which by definition ${ }^{3}$ is one of Appell's hypergeometric functions of two arguments:

$$
F_{4}\left[a, 1+\alpha+\beta-a ; 1+\alpha, 1+\beta ; \frac{t(x-1)}{2}, \frac{t(x+1)}{2}\right] .
$$

Thus the relation developed so far is

$$
\begin{aligned}
F_{4}[a, 1+\alpha+\beta-a ; 1+ & \left.\alpha, 1+\beta ; \frac{t(x-1)}{2}, \frac{t(x+1)}{2}\right] \\
& =\sum_{n=0}^{\infty} \frac{(a)_{n}(1+\alpha+\beta-a)_{n}}{(1+\alpha)_{n}(1+\beta)_{n}} P_{n}^{(\alpha, \beta)} t^{n} .
\end{aligned}
$$

Bailey showed in 1933 and 1934 that the above particular $F_{4}$ could be written as a product of ordinary hypergeometric functions ${ }^{4}$

$$
\begin{aligned}
& F_{4}[a, 1+\alpha+\beta-a ; 1+\alpha, 1+\beta ; u(1-v), v(1-u)] \\
& ={ }_{2} F_{1}(a, 1+\alpha+\beta-a ; 1+\alpha ; u){ }_{2} F_{1}(a, 1+\alpha+\beta-a ; 1+\beta ; v) .
\end{aligned}
$$

This relation is valid for the region about $u=0, v=0$ for which $|u(1-v)|^{1 / 2}+|v(1-u)|^{1 / 2}<1$. Now let $\rho=\left(1-2 x t+t^{2}\right)^{1 / 2}$, using the branch for which $\rho$ becomes 1 at $t=0$, and make the change of variable

$$
v=(1+t-\rho) / 2, \quad u=(1-t-\rho) / 2,
$$

'See W. N. Bailey, Generalized hypergeometric series, Cambridge Tract No. 32, Cambridge University Press, 1935, p. 73.

4 See W. N. Bailey, op. cit. p. 81. 
noting that for a given $x$, a neighborhood of $t=0$ is included under the inequality restriction above. Equations (9), (10), and (11) now combine for the first result of this paper:

$$
\begin{aligned}
& { }_{2} F_{1}\left[\begin{array}{r}
a, 1+\alpha+\beta-a ; \\
1+\alpha ;
\end{array}(1-t-\rho) / 2\right] \\
& { }_{2} F_{1}\left[\begin{array}{c}
a, 1+\alpha+\beta-a ; \\
1+\beta ;(1+t-\rho) / 2
\end{array}\right] \\
& =\sum_{n=0}^{\infty} \frac{(a)_{n}(1+\alpha+\beta-a)_{n}}{(1+\alpha)_{n}(1+\beta)_{n}} P_{n}^{(\alpha, \beta)}(x) t^{n} \text {, } \\
& \text { with } \rho=\left(1-2 x t+t^{2}\right)^{1 / 2} \text {. }
\end{aligned}
$$

Note that the parameter $a$ is arbitrary in (12), except that if $a$ or $(1+\alpha+\beta-a)$ is a nonpositive integer, the series terminates so that only a finite set of the polynomials is involved.

Putting $\alpha=\beta=0$ in (12) gives a generating function for Legendre polynomials $P_{n}(x)$ :

$$
\begin{array}{r}
{ }_{2} F_{1}\left[\begin{array}{c}
a, 1-a ;(1-t-\rho) / 2 \\
1 ;
\end{array}\right]{ }_{2} F_{1}\left[\begin{array}{r}
a, 1-a ;(1+t-\rho) / 2 \\
1 ;
\end{array}\right] \\
=\sum_{n=0}^{\infty} \frac{(a)_{n}(1-a)_{n}}{n ! n !} P_{n}(x) t^{n}, \quad \text { with } \rho=\left(1-2 x t+t^{2}\right)^{1 / 2}
\end{array}
$$

Now consider the terminating cases of (12). Put $a$ equal to the negative integer $-k$ to find:

$$
\begin{aligned}
&{ }_{2} F_{1}\left[\begin{array}{r}
-k, 1+\alpha+\beta+k ; \\
1
\end{array} \quad \begin{array}{r}
\alpha ; \\
1-t-\rho) / 2
\end{array}\right] \\
& \cdot{ }_{2} F_{1}\left[\begin{array}{r}
-k, 1+\alpha+\beta+k ; \\
1+\beta ;
\end{array}(1+t-\rho) / 2\right] \\
&=\sum_{n=0}^{k} \frac{(-k)_{n}(1+\alpha+\beta+k)_{n}}{(1+\alpha)_{n}(1+\beta)_{n}} P_{n}^{(\alpha, \beta)}(x) t^{n} \\
& \rho=\left(1-2 x t+t^{2}\right)^{1 / 2} .
\end{aligned}
$$

Now either (1) or (4) may be converted into

$$
P_{k}^{(\alpha, \beta)}(x)=\frac{(-1)^{k}(1+\beta)_{k}}{k !}{ }_{2} F_{1}\left[\begin{array}{c}
-k, 1+\alpha+\beta+k ; 1+x \\
1+\beta ; 2
\end{array}\right]
$$


Substituting (1) and (15) in (14) yields:

$$
\begin{aligned}
& P_{k}^{(\alpha, \beta)}(t+\rho) P_{k}^{(\alpha, \beta)}(t-\rho) \\
& =\frac{(-1)^{k}(1+\alpha)_{k}(1+\beta)_{k}}{k ! k !} \sum_{n=0}^{k} \frac{(-k)_{n}(1+\alpha+\beta+k)_{n}}{(1+\alpha)_{n}(1+\beta)_{n}} P_{n}^{(\alpha, \beta)}(x) t^{n} \\
& \rho=\left(1-2 x t+t^{2}\right)^{1 / 2} .
\end{aligned}
$$

3. Ultraspherical polynomials. For the ultraspherical polynomials a generating function with an arbitrary parameter results immediately from (12) by placing $\beta=\alpha$ :

$$
\begin{aligned}
{ }_{2} F_{1}\left[\begin{array}{c}
a, 1+2 \alpha-a ;(1-t-\rho) / 2 \\
1+\alpha ;
\end{array}\right] \\
\cdot{ }_{2} F_{1}\left[\begin{array}{c}
a, 1+2 \alpha-a ;(1+t-\rho) / 2 \\
1+\alpha ;
\end{array}\right] \\
=\sum_{n=0}^{\infty} \frac{(a)_{n}(1+2 \alpha-a)_{n}}{(1+\alpha)_{n}(1+\alpha)_{n}} P_{n}^{(\alpha, \alpha)}(x) t^{n}, \quad \rho=\left(1-2 x t+t^{2}\right)^{1 / 2} .
\end{aligned}
$$

However, a separate generating function with arbitrary parameter may be developed for the ultraspherical polynomials. It is

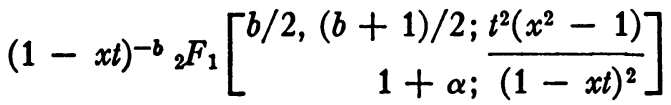

$$
\begin{aligned}
& =\sum_{n=0}^{\infty} \frac{(b)_{n} P_{n}^{(\alpha, \alpha)}(x) t^{n}}{(1+\alpha)_{n}} \text {. }
\end{aligned}
$$

The development here of this generating function makes use of a special form

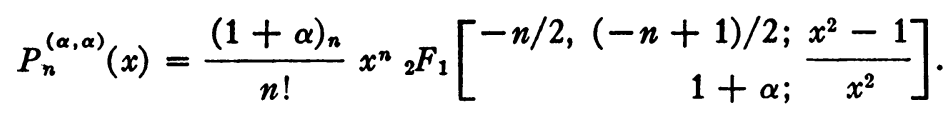

Rewrite equation (19) as

$$
P_{n}^{(\alpha, \alpha)}(x)=(1+\alpha)_{n} \sum_{k=0}^{[n / 2]} \frac{\left(x^{2}-1\right)^{k} x^{n-2 k}}{2^{2 k}(1+\alpha)_{k} k !(n-2 k) !}
$$

and sum as indicated to obtain

$$
\sum_{n=0}^{\infty} \frac{(b)_{n} P_{n}^{(\alpha, \alpha)}(x) t^{n}}{(1+\alpha)_{n}}=\sum_{n=0}^{\infty} \sum_{k=0}^{[n / 2]} \frac{(b)_{n}\left(x^{2}-1\right)^{k} x^{n-2 k} t^{n}}{2^{2 k}(1+\alpha)_{k} k !(n-2 k) !} .
$$


The right-hand side becomes

$$
\sum_{k=0}^{\infty} \sum_{n=0}^{\infty} \frac{(b)_{n+2 k}\left(x^{2}-1\right)^{n} x^{n} t^{n+2 k}}{2^{2 k}(1+\alpha)_{k} k ! n !}
$$

or

$$
\sum_{k=0}^{\infty} \frac{(b)_{2 k}\left(x^{2}-1\right)^{k} t^{2 k}}{4^{k}(1+\alpha)_{k} k !} \sum_{n=0}^{\infty} \frac{(b+2 k)_{n} x^{n} t^{n}}{n !}
$$

or

$$
(1-x t)^{-b}{ }_{2} F_{1}\left[\begin{array}{c}
b / 2,(b+1) / 2 ; \frac{t^{2}\left(x^{2}-1\right)}{(1-x t)^{2}} \\
1+\alpha ; .
\end{array}\right.
$$

Thus there is obtained the generating function (18). The special cases $b=1+\alpha$ and $b=1+2 \alpha$ were previously known. ${ }^{5}$

Another generating function for the Legendre polynomial set may of course be obtained by putting $\alpha=0$ in (18) to get:

$$
(1-x t)^{-b}{ }_{2} F_{1}\left[\begin{array}{c}
b / 2,(b+1) / 2 ; \frac{t^{2}\left(x^{2}-1\right)}{(1-x t)^{2}}
\end{array}\right]=\sum_{n=0}^{\infty} \frac{(b)_{n} P_{n}(x) t^{n}}{n !}
$$

If the arbitrary parameter in (18) is taken as a nonpositive integer, the series terminates and the left side may be expressed as an ultraspherical polynomial itself. This result can be stated as:

$$
\begin{gathered}
\frac{k ! \rho^{k}}{(1+\alpha)_{k}} P_{k}^{(\alpha, \alpha)}\left(\frac{1-x t}{\rho}\right)=\sum_{n=0}^{k} \frac{(-k)_{n} P_{n}^{(\alpha, \alpha)}(x) t^{n}}{(1+\alpha)_{n}} \\
\rho=\left(1-2 x t+t^{2}\right)^{1 / 2} .
\end{gathered}
$$

The emergence of a member of the polynomial set on the left-hand side here naturally tempts one to iterate the process. Details on the iteration may be found on p. 26 of the thesis from which this paper is taken. ${ }^{1}$

4. A set of polynomials which includes ultraspherical and Hermite polynomials as special cases. Consider the polynomial set

$$
{ }_{p+2} F_{q}\left[\begin{array}{r}
-n / 2,(-n+1) / 2, \alpha_{1}, \alpha_{2}, \cdots, \alpha_{p} ; x \\
\beta_{1}, \beta_{2}, \cdots, \beta_{q} ;
\end{array}\right] .
$$

- See Szegö, op. cit. p. 68, and W. Magnus and F. Oberhettinger, Formulas and theorems for the special functions of mathematical physics, Chelsea, 1949, p. 76. In the latter it is necessary to use the knowledge that for the polynomials $C_{n}^{(\alpha)}(x)$ defined there, $C_{n}^{(\alpha)}(x)=\left((2 \alpha)_{n} /(\alpha+1 / 2)_{n}\right) P_{n}^{(\alpha-1 / 2, \alpha-1 / 2)}(x)$. 
By following exactly the same procedure as in $\$ 3$, there can be obtained the generating function

$$
\begin{array}{r}
(1-t)^{-a}{ }_{p+2} F_{q}\left[\begin{array}{r}
a / 2,(a+1) / 2, \alpha_{1}, \alpha_{2}, \cdots, \alpha_{p} ; \\
\beta_{1}, \beta_{2}, \cdots, \beta_{q} ;
\end{array}\right. \\
=\sum_{n=0}^{\infty} \frac{(a)_{n} t^{n}}{n !}{ }_{p+2} F_{q}\left[\begin{array}{r}
-n / 2,(-n+1) / 2, \alpha_{1}, \cdots, \alpha_{p} ; \\
\beta_{1}, \cdots, \beta_{q} ;
\end{array}\right] .
\end{array}
$$

Equation (24) runs parallel to one of Chaundy's results ${ }^{6}$

$$
\begin{aligned}
& (1-t)^{-a}{ }_{p+1} F_{q}\left[\begin{array}{r}
a, \alpha_{1}, \alpha_{2}, \cdots, \alpha_{p} ; \\
\beta_{1}, \beta_{2}, \cdots, \beta_{q} ; 1-t
\end{array}\right] \\
& =\sum_{n=0}^{\infty} \frac{(a)_{n} t^{n}}{n !}{ }_{p+1} F_{q}\left[\begin{array}{r}
-n_{1} \alpha_{1}, \alpha_{2}, \cdots, \alpha_{p} ; x \\
\beta_{1}, \beta_{2}, \cdots, \beta_{q} ;
\end{array}\right],
\end{aligned}
$$

which includes a generating function, equation (32) below, for the Laguerre polynomials

$$
L_{n}^{(\alpha)}(x)=\frac{(1+\alpha)_{n}}{n !}{ }_{1} F_{1}(-n ; \alpha+1 ; x) .
$$

Equations (24) and (25) together give generating functions for two large classes of polynomial sets. Additional generating functions

$$
\begin{aligned}
& e_{p}^{t} F_{q}\left[\begin{array}{r}
\left.\alpha_{1}, \alpha_{2}, \cdots, \alpha_{p} ; \frac{x t^{2}}{4}\right] \\
\beta_{1}, \beta_{2}, \cdots, \beta_{q} ;
\end{array}\right. \\
& =\sum_{n=0}^{\infty} \frac{t^{n}}{n !}{ }_{p+2} F_{q}\left[\begin{array}{r}
-n / 2,(-n+1) / 2, \alpha_{1}, \alpha_{2}, \cdots, \alpha_{p} ; x \\
\beta_{1}, \beta_{2}, \cdots, \beta_{q} ;
\end{array}\right], \\
& e_{p}^{t} F_{q}\left[\begin{array}{r}
\alpha_{1}, \alpha_{2}, \cdots, \alpha_{p} ; \\
\beta_{1}, \beta_{2}, \cdots, \beta_{q} ;
\end{array}\right] \\
& =\sum_{n=0}^{\infty} \frac{t^{n}}{n !}{ }_{p+1} F_{q}\left[\begin{array}{r}
-n, \alpha_{1}, \alpha_{2}, \cdots, \alpha_{p} ; x \\
\beta_{1}, \beta_{2}, \cdots, \beta_{q} ;
\end{array}\right]
\end{aligned}
$$

may be obtained immediately for each class by omitting the parameter $a$ before summing.

Interesting special cases may be obtained from these general sets. In equation (24) omit the parameters $\alpha_{1}, \alpha_{2}, \cdots, \alpha_{p}$, take one denominator parameter as $1+\alpha$, replace $x$ by $\left(x^{2}-1\right) / x^{2}$ and then $t$ by $t x$. The result is equation (18) on ultraspherical polynomials.

' See T. W. Chaundy, An extension of the hypergeometric function, Quart. J. Math. Oxford Ser. vol. 14 (1943) p. 62. 
To obtain a generating function for Hermite polynomials from (24), omit all $\alpha$ 's and $\beta$ 's, replace $x$ by $\left(-1 / x^{2}\right)$ and then $t$ by $2 x t$. This gives

$$
(1-2 x t)^{-a} F_{0}\left[a / 2,(a+1) / 2 ;-; \frac{-4 t^{2}}{(1-2 x t)^{2}}\right]
$$

$$
\approx \sum_{n=0}^{\infty} \frac{(a)_{n} H_{n}(x) t^{n}}{n !}
$$

which is a divergent ${ }^{7}$ generating function for Hermite polynomials already known in different form for the special case $a=1 .^{8}$

5. A peculiar result on Hermite polynomials. The nonstandard type of generating function

$$
\left(1+4 t^{2}\right)^{-3 / 2}\left(1+2 x t+4 t^{2}\right) \exp \left(\frac{4 x^{2} t^{2}}{1+4 t^{2}}\right)=\sum_{n=0}^{\infty} \frac{H_{n}(x) t^{n}}{[n / 2] !}
$$

has been known for some time. ${ }^{9}$ A generalization of (29) follows.

It is well known that

$$
H_{2 n}(x)=(-4)^{n} n ! L_{n}^{(-1 / 2)}\left(x^{2}\right), \quad H_{2 n+1}(x)=2 x(-4)^{n} n ! L_{n}^{(1 / 2)}\left(x^{2}\right) .
$$

Insert these in the identity

$$
\begin{aligned}
\sum_{n=0}^{\infty} \frac{H_{2 n}(x)(c)_{n} t^{2 n}}{n !(d)_{n}}+\sum_{n=0}^{\infty} \frac{H_{2 n+1}(x)(c)_{n} t^{2 n+1}}{n !(d)_{n}} & \\
= & \sum_{n=0}^{\infty} \frac{H_{n}(x)(c)_{[n / 2]} t^{n}}{[n / 2] !(d)_{[n / 2]}}
\end{aligned}
$$

to obtain

$$
\begin{aligned}
\sum_{n=0}^{\infty} \frac{(-4)^{n}(c)_{n} L_{n}^{(-1 / 2)}\left(x^{2}\right) t^{2 n}}{(d)_{n}}+2 x t \sum_{n=0}^{\infty} \frac{(-4)^{n} t^{2 n}(c)_{n} L_{n}^{(1 / 2)}\left(x^{2}\right)}{(d)_{n}} \\
=\sum_{n=0}^{\infty} \frac{H_{n}(x)(c)_{[n / 2]} t^{n}}{[n / 2] !(d)_{[n / 2]}}
\end{aligned}
$$

${ }_{7}$ The series represented by the ${ }_{2} F_{0}$ on the left side of (28) diverges unless $a$ is a nonpositive integer. Equation (28) implies a formal relation between the coefficients of $t^{n}$ on each side. A precise definition is given on p. 40 of the author's dissertation.

${ }^{8}$ See C. Truesdell, On the functional equation $\partial F(z, \alpha) / \partial z=F(z, \alpha+1)$, Proc. Nat. Acad. Sci. U.S.A. vol. 33 (1947) p. 88.

$\checkmark$ This generating function and reference to Doetsch are given in Szegö, op. cit. p. 371. 
Now for the special case of Laguerre polynomials, Chaundy's equation (25) reduces to

$$
(1-t)^{-a}{ }_{1} F_{1}\left(a ; 1+\alpha ; \frac{-x t}{1-t}\right)=\sum_{n=0}^{\infty} \frac{(a)_{n} L_{n}^{(\alpha)}(x) t^{n}}{(1+\alpha)_{n}}
$$

Take $d=1 / 2$ in (31). Then use (32) to find for the first term on the left-hand side of (31)

$$
\left(1+4 t^{2}\right)^{-c} F_{1}\left(c ; 1 / 2 ; \frac{4 x^{2} t^{2}}{1+4 t^{2}}\right)
$$

and for the second term on the left-hand side of (31)

$$
(2 x t)(1+\theta)\left[\left(1+4 t^{2}\right)^{-c}{ }_{1} F_{1}\left(c ; 3 / 2 ; \frac{4 x^{2} t^{2}}{1+4 t^{2}}\right)\right]
$$

where $\theta \equiv t(d / d t)$. Incorporating these last results in (31) and carrying out the operation by $\theta$ yields:

$$
\begin{aligned}
&\left(1+4 t^{2}\right)^{-c}{ }_{1} F_{1}(c ;\left.\frac{1}{2} ; \frac{4 x^{2} t^{2}}{1+4 t^{2}}\right) \\
&+\frac{32 c t^{3} x^{3}}{3\left(1+4 t^{2}\right)^{c+2}}{ }_{1} F_{1}\left(\begin{array}{l}
\left.c+1 ; \frac{4 x^{2} t^{2}}{1+4 t^{2}}\right) \\
5 / 2 ;
\end{array}\right. \\
& \quad+\frac{2 x t\left(1+4 t^{2}-8 c t^{2}\right)}{\left(1+4 t^{2}\right)^{c+1}}{ }_{1} F_{1}\left(c ; 3 / 2 ; \frac{4 x^{2} t^{2}}{1+4 t^{2}}\right) \\
&=\sum_{n=0}^{\infty} \frac{H_{n}(x)(c)_{[n / 2]} t^{n}}{[n / 2] !(1 / 2)_{[n / 2]}}
\end{aligned}
$$

which is the promised generalization of (29). It contains the arbitrary parameter $c$, and reduces to (29) for $c=1 / 2$. Identification of this reduction with (29) involves using the easily proved relation

$$
\begin{aligned}
\frac{z}{a+1}{ }_{1} F_{1}(a+1 ; a+2 ; z)+{ }_{1} F_{1}(a ; a+1 ; z) & =e^{z}, \\
a & \neq-1,-2, \cdots .
\end{aligned}
$$

The University of Michigan 